\title{
An Orthogonal Waveform for Fully Polarimetric MIMO-SAR
}

\author{
Tobias Rommel, Marwan Younis, Gerhard Krieger \\ German Aerospace Centre (DLR) \\ Microwaves and Radar Institute \\ Germany, Muenchener Strasse 20, 82234 Wessling \\ Email: tobias.rommel@dlr.de
}

\begin{abstract}
Synthetic aperture radar (SAR) with multiple transmit and receive channels (MIMO-SAR) has a higher flexibility and an improved efficiency compared to a conventional SAR system with a single channel. The multiple receive channels can be used, among other things, to increase the swath width at constant azimuth resolution or to suppress spatial interferences. However, multiple transmit channels, which transmit simultaneously in the same frequency band provide currently a challenge. Therefore, in this paper a modified chirp waveform is introduced which extends in combination with digital beam-forming (DBF) on receive the orthogonality condition to another degree of freedom, thus allowing in theory perfect orthogonality. Furthermore, the hardware design of the MIMO-Radar Demonstrator, a multichannel measurement system for radar and SAR applications is depicted. The shown measurement result demonstrates the possibility of obtaining all four parameters of the scattering matrix at the same time by transmitting orthogonal waveforms in different polarizations.
\end{abstract}

\section{INTRODUCTION}

In the future, synthetic aperture radar (SAR) must meet higher requirements in terms of resolution, swath width and flexibility. This is due to the enormous success of the current satellite SAR missions (TanDEM-X, Radarsat-2, etc.) and the many users who demand the on-going development of the SAR technology [1], [2]. A consideration of various on-going studies shows that SAR sensors with multiple transmit and receive channels and digital beam-forming (DBF) capabilities have promising properties. Present limitations are mainly caused by the well known contradicting requirement of SAR, where the swath-width limits the resolution in azimuth and vice versa. To give a concrete example, the satellite TerraSAR$\mathrm{X}$ can achieve in Stripmap mode and a swath-width of $30 \mathrm{~km}$ only a maximum resolution of $3 \mathrm{~m}$. Since the TanDEM-X mission needs for a global coverage approximately one year, the follow-on sensors, like Tandem-L should be designed for a swath width of $350 \mathrm{~km}$ with a resolution of $3 \mathrm{~m}$ in a fullpolarized mode. Compared to state-of-the-art, this results in an increase of the performance by the factor of 23 .

Further examples are the observation of several areas with different resolutions (Hybrid-SAR modes) or as depicted in section IV, to perform Quad-Polarization. Recently, a groundbased prototype of these future radar systems has been developed for the verification of such innovative modes and is available at the German Aerospace Centre (DLR).
The paper starts with a discussion of the necessary condition for a reflectivity map free of ambiguities. Afterwards the new transmit waveform with perfect orthogonal properties for point and surface targets is introduced and it's crucial limitation is demonstrated. The next section gives an explanation how this limitation can be circumvented by an innovative DBF process. Then, in section III the MIMO-Radar Demonstrator is described, which is used for the verification of the waveforms. The obtained fully polarimetric measurement results are depicted in section IV. The paper concludes with a summary of the discussed results.

\section{ORThOGONAL SignAls FOR IMAGING RADAR AND MIMO-SAR}

\section{A. The Need for MIMO}

A problem will arise, if several user demand for acquisitions in different areas at the same time (e.g. catastrophes). As a solution, several authors suggest to use multiple transmit and receive channels (MIMO) to overcome this contradiction and to enhance the capabilities of the sensor. With so called Hybrid-SAR modes it becomes possible to observe different areas at the same time, where the sensor illuminates with multiple transmit channels different areas on the Earth and the receivers record the backscattered data simultaneously.

In particular, polarimetry is gaining popularity because the obtained images provide conclusions of the dielectric properties of the scatterers. A separation between man-made and natural surfaces becomes then easily possible by color-coding. To obtain all four parameters of the scattering matrix (Quadpolarization), two independent measurements have to be taken. At the current state, the sensor has to transmit the signals in an interleaved mode in horizontal and vertical polarization successively, whereas both polarizations are received simultaneously. Thus, to fulfill the Nyquist theorem in azimuth, the $P R F$ has to be increased by the factor of 2 to keep the effective sampling rate the same. Finally, this will reduce the swath width. Consequently the simultaneous acquisition of the whole scattering matrix would be a tremendous advantage. With the method proposed in this paper, both polarizations can be transmitted at the same time, whereas the whole scattering matrix will be obtained later in the receiver with an innovative beam-forming algorithm. As a result, the swath width can be kept constant. 


\section{B. Orthogonal Condition}

The MIMO-SAR system fully exploits its degrees of freedom by simultaneously activating multiple transmit antennas in the same frequency band. To obtain orthogonality for these signals, several authors have suggested to simultaneously transmit mutually orthogonal waveforms. However it was clearly shown in [7] that this only works for small swath widths with a decreased signal to noise ratio. The reason for this is that the orthogonality condition between the transmit signals $s_{\mathrm{a}}$ and $s_{\mathrm{b}}$ is typically formulated as:

$$
\int s_{\mathrm{a}}(t) \cdot s_{\mathrm{b}}^{*}(t) \cdot d t=0 \quad \text { for } \quad \mathrm{a} \neq \mathrm{b},
$$

which is valid for a dedicated point target. This condition is sufficient for most of the radar applications where a few pointlike targets are detected, which are within different resolution cells. But it may pose a challenge in a distributed scatterer scenario when there is a time shift between $s_{\mathrm{a}}$ and $s_{\mathrm{b}}$. By observing large swaths with an imaging radar or a SAR sensor the long echoes are already filling a huge part of the timefrequency plane. The echoes of the different transmit signals will then overlap and it becomes impossible to separate them later in the receiver. Thus, the condition to achieve is to ensure orthogonality for arbitrary shifts $\tau$ :

$$
\int s_{\mathrm{a}}(t) \cdot s_{\mathrm{b}}^{*}(t-\tau) \cdot d t=0 \quad \forall \quad \tau \in \mathbb{R}, \mathrm{a} \neq \mathrm{b} .
$$

\section{Short-Term Shift-Orthogonal Waveform}

To avoid an overlap and thus ambiguities of the transmitted signals a new, partly orthogonal waveform has been proposed by Krieger in [8] and later introduced in [6], [7]. For two transmitters, the basic idea behind this approach can be expressed by a restricted orthogonality:

$$
\begin{gathered}
\int s_{\mathrm{a}}(t) \cdot s_{\mathrm{b}}^{*}(t-\tau) \cdot d t=0 \\
\forall \quad \tau \in\left[-\frac{\tau_{\text {pulse }}}{2}, \frac{\tau_{\text {pulse }}}{2}\right] \text { and } \mathrm{a} \neq \mathrm{b},
\end{gathered}
$$

where $\tau_{\text {pulse }}$ is the pulse duration. Now, the two different transmit signals $s_{\mathrm{a}}$ and $s_{\mathrm{b}}$ have only orthogonal properties for a subset of signal shifts. In the example chosen here, this time interval is limited by half of the pulse length. This allows a perfect separation of the echoes of neighboring objects for a signal delay $\tau<\tau_{\text {pulse }}$. A simple example of a suitable signal is the simultaneous transmission of shifted frequency ramps. Fig. 1 shows the signals in the time-frequency plane.

Transmit channel Tx1 is fed with a normal up-chirp, while transmit channel Tx2 is fed with a shifted up-chirp (also called Short-Term Shift-Orthogonal Waveform). It is clear that the signals do not overlap for signal propagation times shorter than $c_{0} \cdot \tau_{\text {pulse }} / 4$, where $c_{0}$ is the propagation speed of light. The factor of 4 is due to the two way propagation radartarget + target-radar and the $50 \%$ shift of the pulse. However, if a larger swath is considered, the signal propagation time

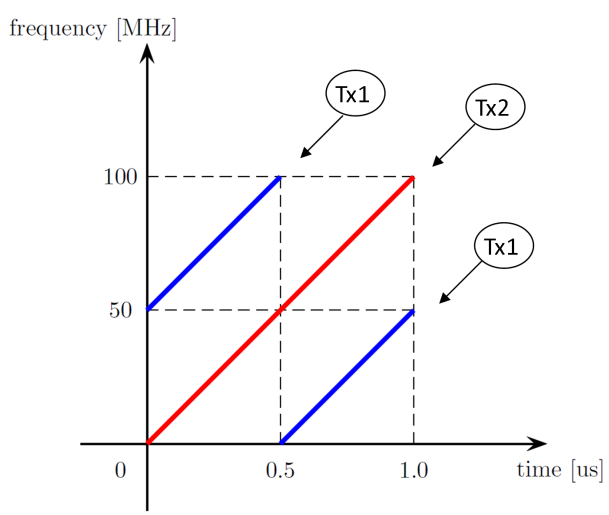

Fig. 1. Short-Term Shift-Orthogonal Waveform in the time-frequency plane.

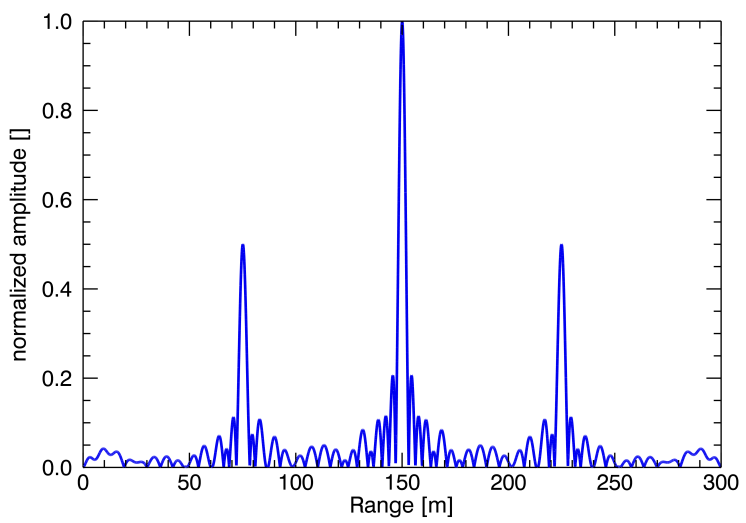

Fig. 2. Impulse response of a point target at $150 \mathrm{~m}$.

increases and the time-frequency plane is completely filled with the echoes, so that the two signals will finally overlap. In this case, they are no longer orthogonal, which represents the limitation described in Eq. 3 to this waveform.

The scheme of chirp signals can also be expanded easily to more than two transmit channels by splitting it into $N_{\mathrm{Tx}}$ parts, where $N_{\text {Tx }}$ is the number of transmit channels. Fig. 2 shows the impulse response of a simulated point target at a distance of $150 \mathrm{~m}$. With the chosen pulse width of $\tau_{\text {pulse }}=$ $1 \mu \mathrm{s}$, ambiguities appear at a distance of $\pm c_{0} \cdot \tau_{\text {pulse }} / 4(75 \mathrm{~m}$ and $225 \mathrm{~m}$ ). These result from the convolution with the other transmit channel. Since an up-chirp is used for $s_{\mathrm{a}}$, all signal characteristics compared to a conventional pulsed radar chirp are identical. The phase shift in the middle of the pulse of channel 2 is assumed to be negligible for large time-bandwidth products.

Because usually the swath width exceeds the pulse length, this type of waveforms will also cause ambiguities. Therefore, in the next section a solution to overcome this limitation with the aid of digital beam-forming on receive is described.

\section{Suppression of Ambiguities with an Extended SCORE Algorithm}

To suppress the ambiguities which have been described in the last section, the use of digital beam-forming on receive in 


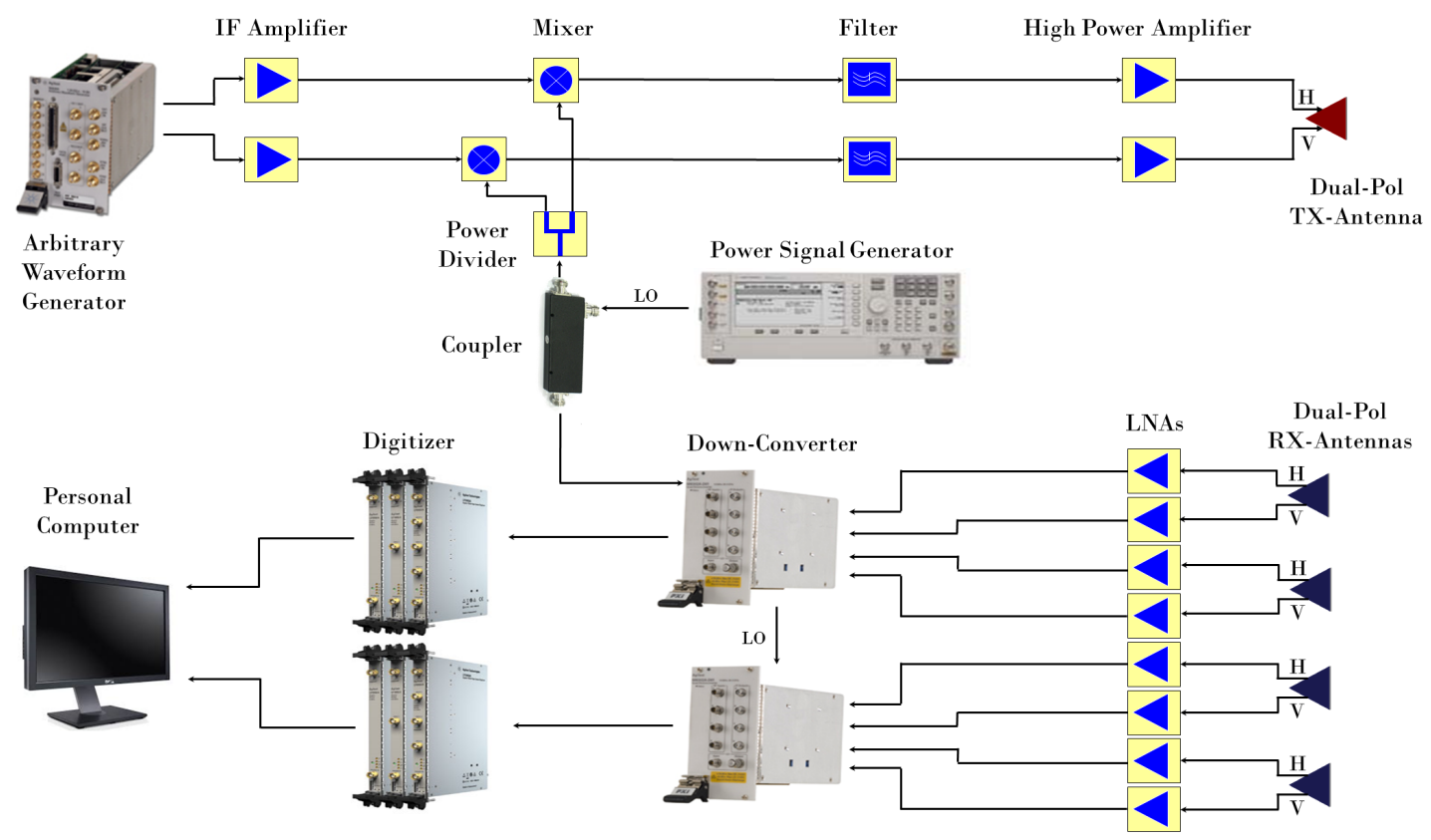

Fig. 3. Simplified block diagram of the MIMO-Radar Demonstrator.

the elevation plane for each instance of the moving platform is proposed. Due to the property of the side-looking radar (compare Fig. 4), each point in the range direction is seen from a different angle of incidence:

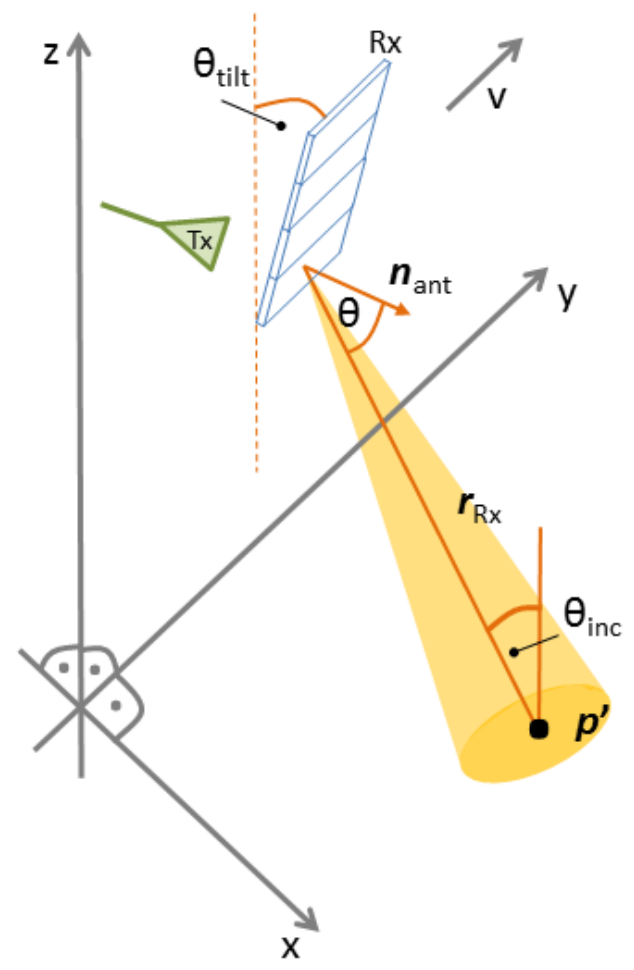

Fig. 4. Side-looking geometry of the radar moving with the velocity $v$ in the y-direction.

$$
\Theta=\arccos \left(\frac{\boldsymbol{n}_{\mathrm{ant}}^{\mathrm{T}} \boldsymbol{r}_{\mathrm{Rx}}}{\left\|\boldsymbol{n}_{\mathrm{ant}}\right\| \cdot\left\|\boldsymbol{r}_{\mathrm{Rx}}\right\|}\right),
$$

where $\boldsymbol{n}_{\text {ant }}$ denotes the antenna normal vector, $\boldsymbol{r}_{\mathrm{Rx}}$ is the time varying distance between the target and the antenna and $\|$.$\| denotes the euclidean norm. With the known relation of$ frequency, time, and angle of arrival, it is possible to distinguish between the individual transmit signals through beamforming on receive. In the proposed method (also known as SCORE, Scan-On-Receive) [3] a broad swath on the ground is illuminated with the small apertures of the transmit antennas. On receive, all receive elements of the array antenna are activated and weighted with phase shifts so that there is a narrow directional beam, which follows the expected response on the ground. For an uniform linear array (ULA) with an antenna element separation of $d$ and the signal wavelength $\lambda_{0}$ the phase shift $\Delta \varphi_{i}$ required to steer the beam maximum to $\Theta$ can be expressed by:

$$
\Delta \varphi_{i}(\Theta)=\frac{2 \pi}{\lambda_{0}} d(i-1) \cdot \sin (\Theta),
$$

where the index $i$ corresponds to the $i^{\text {th }}$ receive channel. These phase shifts can then be written at a time-variant steering vector:

$$
\boldsymbol{a}(\Theta)=\boldsymbol{a}(\tau)=\left[e^{-\mathrm{j} \Delta \varphi_{1}}, \ldots, e^{-\mathrm{j} \Delta \varphi_{N_{\mathrm{Rx}}}}\right]^{T} .
$$

To form a narrow beam in the digital processor, the corresponding weight vector $\boldsymbol{w}(\tau)$ for multiplication with the received raw data is obtained by the hermitian ${ }^{\mathrm{H}}$ of the steering vector:

$$
\boldsymbol{w}(\tau)=\boldsymbol{a}^{\mathrm{H}}(\tau)
$$


This multiplication of the raw data for each receive channel with the weighting coefficient $\boldsymbol{w}(\tau)$, is also called digital beam-forming.

Because each transmitted pulse has a certain duration, on receive, the echo at the phase center arrives simultaneously from all points located between the leading and the lagging edge of the pulse on the ground. This effect is also called pulse extension loss (PEL) [11]. To overcome this loss an additional correction factor in frequency domain has to be considered:

$$
\boldsymbol{H}_{i}=\boldsymbol{s}_{j}^{*}(f) \cdot \exp \left(\frac{-\mathrm{j} 2 \pi d(i-1) \cdot \sin (\Theta)}{c_{0}} f\right),
$$

where the index $j$ corresponds to the $j^{\text {th }}$ transmitted waveform. Because the tensor $\boldsymbol{H}$ is not only dependent of time and angle of arrival, but also of the transmitted signal, $\boldsymbol{H}$ can be seen as a matched filter. Thus, in the next step the waveforms will already be separated and a range compression is directly applied. This phase shift can be implemented in frequency domain as a multiplication with the received raw data $\boldsymbol{u}$ and $\boldsymbol{H}_{i}$ :

$$
\boldsymbol{k}_{i, j}(\tau)=\mathcal{F}^{-1}\left\{\mathcal{F}\left\{\boldsymbol{u}_{i}\right\} \cdot \boldsymbol{H}_{i}\right\},
$$

with $\mathcal{F}$ and $\mathcal{F}^{-1}$ denoting the Fourier Transform and the Inverse Fourier Transform, respectively. Finally, the output $\boldsymbol{y}_{j}$ of the beamformer is expressed by:

$$
\boldsymbol{y}_{j}=\boldsymbol{w}(\tau) \cdot \boldsymbol{k}_{i, j}(\tau)
$$

This innovative transmit-waveform adaptive SCORE algorithm is performed for a SAR for each sample in azimuth in time, frequency and space. After a coherent combination of all the receive channels in elevation, other processing steps for typical SAR can be applied. The main advantage of this waveform and processing technique is that the transmitted signals can be completely separated and thus allowing an additional degree of freedom for various operation modes of next generation SAR sensors.

\section{MIMO-RADAR DEMONSTRATOR}

The architecture of the modular, reconfigurable radar with multiple transmit and receive channels is shown in Fig. 3. The purpose of this system is to test and verify new techniques and concepts for future satellite-based remote sensing missions. The configuration described in this paper, operates in X-band with two transmit and eight receive channels. At present, the radar can also be operated in the P-band (up to $500 \mathrm{MHz}$ ). The modular design in 19" form allows great flexibility of system changes and extensions. As depicted in Fig. 3, the desired transmit signals are generated in an arbitrary waveform generator having a bandwidth of $\max .500 \mathrm{MHz}$. Then they are amplified and up-converted to the carrier frequency. Before transmission, the lower sidebands and the carrier signals are suppressed by bandpass filters and the remaining signals are amplified with high-power amplifiers. Afterwards they are transmitted with a dual-polarized patch antenna, whereas each channel is connected to a different polarization. In the receive chain, the echoes are received simultaneously by all Rx antennas in horizontal and vertical polarization, amplified with lownoise amplifiers, down-converted and finally sampled by AD converters. The independent data recording for each receive channel allows the subsequent formation of different arbitrary beam patterns of the antenna in the processing. The additional information can be used to suppress spatial ambiguities in the signal, to increase the gain of the receive antenna, or to suppress spatial interferences. Basic system properties have been already presented in [10]. In comparison to a radar system with a single channel, stationary two-dimensional radar imaging, improved azimuth resolution and an improved signal to noise ratio have been verified successfully. These measurements were used as the first step towards high-resolution SAR imaging with large swath widths.

\section{Measurement Results}

For the demonstration and verification of the Short-Term Shift-Orthogonal Waveforms in combination with polarization, an outdoor measurement of the target localization in the range direction was conducted with the MIMO-Radar Demonstrator. As already presented in former publications [6], [10], the spatial filtering and proper matched filtering of the proposed waveforms with this system is working. Hereupon we present the first result of Quad-polarization in this paper, where the Radar was placed on top of a roof in a height of $12.7 \mathrm{~m}$ and two dihedral corner reflectors with a ground plate of the size $a \cdot b=0.4 \mathrm{~m} \cdot 0.3 \mathrm{~m}=0.12 \mathrm{~m}^{2}(\mathrm{RCS}=26 \mathrm{dBsm})$ were placed on a meadow at ground range distances of $46.4 \mathrm{~m}$ and $55.3 \mathrm{~m}$, respectively. According to the polarimetric scattering matrix of a dihedral corner reflector

$$
\boldsymbol{S}(\alpha)=\sqrt{\frac{\sigma}{4 \pi}}\left[\begin{array}{cc}
-\cos (2 \alpha) & \sin (2 \alpha) \\
\sin (2 \alpha) & \cos (2 \alpha)
\end{array}\right]
$$

with

$$
\sigma=\frac{8 \pi a^{2} b^{2}}{\lambda^{2}}
$$

a rotation of the reflector in the $\mathrm{y}$-z-plane by the angle $\alpha$ changes the scattering behavior. The near corner reflector was rotated by $\alpha=-45^{\circ}$, whereas the far corner was orientated with $\alpha=0^{\circ}$. Using this constellation, the near reflector should only be visible in the cross-polar components $H V$ and $V H$. The reflector at $39.6 \mathrm{~m}$ can then only be seen in the $H H$ and $V V$ polarized data sets.

The two orthogonal transmit signals with a pulse duration of $\tau_{\text {pulse }}=0.4 \mu \mathrm{s}$ and a bandwidth of $B=300 \mathrm{MHz}$ (corresponding to a range resolution of $0.5 \mathrm{~m}$ ) were transmitted simultaneously at a center frequency of $9.58 \mathrm{GHz}$ in the horizontal and vertical polarization with a single antenna. This pulse duration was chosen so that signal ambiguities will occur within the scene of interest if the extended SCORE algorithm described in section IID fails. With the known geometry of the setup, signal ambiguities were then expected at $12.9 \mathrm{~m}, 23.5 \mathrm{~m}, 77.1 \mathrm{~m}$, and $85.8 \mathrm{~m}$ on the ground. On 
receive, four dual polarized antennas with an antenna element spacing of $1.6 \lambda$ were arranged in the elevation plane with a tilt angle of $\Theta_{\text {tilt }}=20^{\circ}$. The measurement results for the cross- and co-polar data sets are depicted in Fig. 5 and Fig. 6. Possible signal ambiguities were suppressed beyond the response of the meadow and are not traceable anymore. The near target is visible as expected in the cross-polar data and was suppressed by $20.8 \mathrm{~dB}$ in the co-polar result. As expected, the far target is visible in the co-polar data and was suppressed by $18.5 \mathrm{~dB}$ in the cross-polar result. The imperfect suppression and disagreement between the two targets result primarily from a misalignment of the targets. Furthermore, the antenna radiation patterns with a difference of the gain in the horizontal and vertical polarization of $1 \mathrm{~dB}$ at the maximum were not considered.

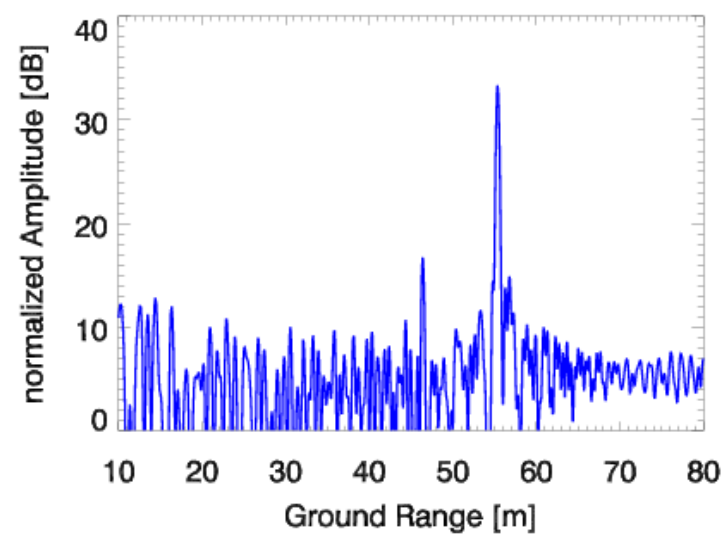

Fig. 5. Impulse response of the Co-polar data sets $|H H|+|V V|$.

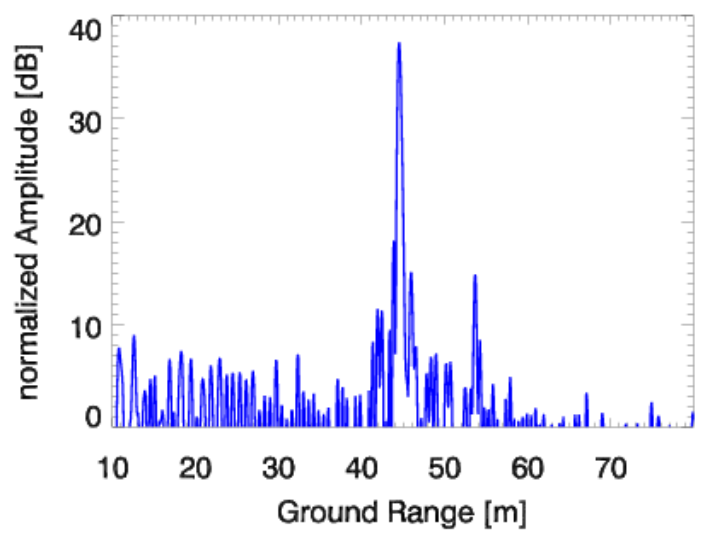

Fig. 6. Impulse response of the Cross-polar data sets $|H V|+|V H|$.

Nevertheless this preliminary measurement result proves that it becomes possible to obtain all four components of the scattering matrix $(H H, V V, H V, V H)$ at the same time by applying the extended SCORE algorithm in combination with Short-Term Shift-Orthogonal Waveforms. Since orthogonality for each transmitted pulse exist, for each polarization further standard SAR processing can be applied without any degradation of the swath-width, resolution or SNR. This measurement result proved that the Short-Term Shift-Orthogonal Waveforms can be used for MIMO-SAR observing large swaths with high resolution in a full-polarized acquisition mode.

\section{CONCLUSION}

In this paper, a new form of transmit signal for MIMO-SAR and imaging radar systems was introduced with a description of its fundamental limitation. It was described how this limitation can be overcome by digital beam-forming on receive. The innovative extension of the classical SCORE algorithm makes it possible to apply a filtering in space, time and frequency. Finally, an overview of the structure of the MIMORadar Demonstrator was given and the theoretical aspects were verified by a full-polarized measurement.

The results, which were presented in this paper, are a first step in the development of future satellite-based Earth observation missions. Other innovative concepts, such as multichannel calibration, DBF with reflector antennas, Hybrid-SAR modes and nonlinear pulse repetition frequencies are already in progress.

\section{REFERENCES}

[1] G. Krieger, A. Moreira, H. Fiedler, I. Hajnsek, M. Werner, M. Younis and M. Zink, TanDEM-X: A Satellite Formation for High-Resolution SAR Interferometry, IEEE TRANSACTIONS ON GEOSCIENCE AND REMOTE SENSING Vol. 45, November 2007, pp. 3317-3341.

[2] A. A. Thompson, D. MacDonald, A. Luscombe, K. James and P. Fox, RADARSAT-2 Mission Status: Capabilities Demonstrated and Image Quality Achieved, Proc. EUSAR 2008, Friedrichshafen, Germany, June 2008.

[3] M. Süss and W. Wiesbeck, Side-Looking Synthetic Aperture Radar System, European Patent Application, EP 1241487 A1, Sept. 18, 2002.

[4] D. Christallini, D. Patina and P. Lombardo, Exploiting MIMO SAR Potentialities With Efficient Cross-Track Constellation Configurations for Improved Range Resolution, IEEE TRANSACTIONS ON GEOSCIENCE AND REMOTE SENSING Vol. 49, January 2011, pp. 38-52.

[5] E. Fishler, A. Haimovich, R. Blum, D. Chizhik, L. Cimini and R. Valenzuela, MIMO RADAR: An Idea Whose Time Has Come, Proc. IEEE Radar Conference, Philadelphia, P, USA, April 2004.

[6] T. Rommel, A. Patyuchenko, P. Laskowski, M. Younis and G. Krieger, An Orthogonal Waveform Scheme for Imaging MIMO-Radar Applications, Proc. IRS 2013, Dresden, Germany, June 2013, pp. 917-922.

[7] G. Krieger, MIMO-SAR: Opportunities and Pitfalls, IEEE TRANSACTIONS ON GEOSCIENCE AND REMOTE SENSING, 2013.

[8] G. Krieger, M. Younis, S. Huber, F. Bordoni, A. Patyuchenko, J. Kim, P. Laskowski, T. Rommel, M. Villano, F. Lopez Dekker and A. Moreira, Digital Beamforming and MIMO SAR - Review and New Concepts, Proc. IGARSS 2012, Munich, Germany, pp. 1533-1536, 2012.

[9] J. Kim, M. Younis, A. Moreira and W. Wiesbeck, A Novel OFDM Waveform for Fully Polarimetric SAR Data Acquisition, Proc. EUSAR 2010, Aachen, Germany, 2010.

[10] A. Patyuchenko, T. Rommel, P. Laskowski, M. Younis, G. Krieger, Digital Beam-Forming Reconfigurable Radar System Demonstrator, Proc. IGARSS 2012, Munich, Germany, pp. 1541-1544.

[11] M. Younis, S. Huber, A. Patyuchenko, F. Bordoni and G. Krieger, Performance Comparison of Reflector- and Antenna Based Digital BeamForming SAR, International Journal of Antennas and Propagation Vol. 2009, pp. 1-13. 